\title{
Catheter ablation of drug resistant supraventricular tachycardia in neonates and infants
}

\author{
Celal Akdeniz, Yakup Ergul, Neslihan Kiplapinar, Volkan Tuzcu \\ Mehmet Akif Ersoy Cardiovascular Research and Training Hospital, Istanbul, Turkey
}

\begin{abstract}
Background: The aim of this study was to evaluate the indications, results and complications of radiofrequency ablation (RFA) and transcatheter cryoablation (TCA) in neonates and infants with incessant drug-resistant supraventricular tachycardia (SVT).
\end{abstract}

Methods: Out of 225 patients who underwent RFA and TCA at our center between January 2010 and February 2012, 5 patients under the age of 1 (4 male, 1 female) were evaluated. The indication for RFA/TCA was recurrent hemodynamically compromising drug-resistant SVT.

Results: Over a 2-year period, 6 ablation procedures were performed in 5 patients. Average patient age was $3.3 \pm 3.9$ months (12 days - 9.5 months); average patient weight was $5.4 \pm 2.2 \mathrm{~kg}(3.5-9 \mathrm{~kg})$. One patient had ventricular septal defect, 1 had corrected transposition of great arteries, ventricular septal defect, right ventricular hypoplasia and pulmonary hypertension, while 3 had only patent foramen ovale. Electrophysiology study showed 1 accessory pathway in each patient (right posteroseptal in 2, left posteroseptal in 2 and left lateral in 1). The pathway was manifest in 1 patient with Wolff-Parkinson-White syndrome (WPW) and concealed in the rest. Two of the concealed pathways had slow conduction time and decremental properties (the permanent form of junctional reciprocating tachycardia). Two patients underwent TCA and 3-RFA, with an acute success rate of 100\%. In the first week after the procedure, the patient with the complex cardiac anomaly and WPW developed recurrence and underwent ablation again. Four of the procedures were carried out using an electroanatomic mapping system besides fluoroscopy. Average procedure time was 167 min (100-234); fluoroscopy time was 8.2 min (0.7-19.7). None of the patients developed major complications. After the average follow-up period of 6.5 months (3-18), all patients were symptom-free without medication.

Conclusions: RFA and TCA can be performed successfully in neonates and infants with incessant medically refractory SVT. (Cardiol J 2013; 20, 3: 241-246)

Key words: catheter ablation, supraventricular tachycardia, neonate, infant

\section{Introduction}

Supraventricular tachycardia (SVT) is the most common type of tachyarrhythmia seen in pediatric patients, and accounts for more than
$90 \%$ of pediatric arrhythmias [1]. Symptoms vary according to age, duration of tachycardia and heart rate; neonates and infants can be admitted with congestive heart failure findings [1]. Although SVT in infants can be managed medically in most

Address for correspondence: Volkan Tuzcu, MD, Professor, Director of Electrophysiology, Division of Pediatric Cardiology, Mehmet Akif Ersoy Research and Training Hospital, Halkali, Istanbul, Turkey, tel: +90 2126922000 (ext. 1446), fax: +90212 47194 94; e-mail: vtuzcu@gmail.com 
cases and tends to spontaneously resolve within 1 or 2 years, there is occasionally need for ablative therapy [1-3].

Radiofrequency ablation (RFA) has been demonstrated to be safe and effective in pediatric patients. In many centers, catheter ablation has become standard treatment of SVT in adolescents with symptomatic tachycardia [4, 5]. Although RFA has been used to treat SVT in younger patients and infants, it has generally been reserved for cases of life-threatening medically refractory tachyarrhythmia $[3,6,7]$. Due to low patient age and weight, concomitant congenital heart diseases and excessive application of radiofrequency (RF) energy, RFA has been linked to increased morbidity and mortality [4, 8, 9]. Restrictions have also been placed on the use of RFA in smaller children since RFA lesions have been shown to have a progressing nature in animal studies [10], in addition to causing coronary ischemia and venous complications in infants [11]. In the recent years, use of transcatheter cryoablation (TCA) in children weighing less than $15 \mathrm{~kg}$ has been shown to be relatively complication-free [12]. This report summarizes our experience in using RFA and TCA to treat medically refractory SVT in neonates and infants. It is also the first study in our country to report on the use of catheter ablation in pediatric patients less than 1 year of age.

\section{Methods}

From January 2010 to February 2012, 225 SVT patients underwent RFA and/or TCA at our center. Five patients were under 1 year of age (4 male, 1 female). The indication for RFA/TCA in these patients was recurrent hemodynamically compromising SVT that was resistant to medical management, including amiodarone, esmolol, propafenon and a combination of class Ic and III agents. The following patient data were obtained from hospital records: age, gender, weight, the presence of structural heart disease, SVT mechanism, accessory pathway location, catheter approach, SVT cycle length, number of RFA/TCA applications, total RFA/TCA time, average and maximum RFA/TCA temperatures, total procedure time, short-term success, complications, and follow-up.

\section{Electrophysiological study}

Informed consent was obtained from all parents prior to the procedures. Electrophysiology studies were carried out under general anaesthesia with the patients intubated. Hemodynamically compromised patients were observed in intensive care conditions, and those with left ventricular dysfunction were started on inotropic agents like dopamine, milrinone and adrenaline before the procedure began. In all patients, lines were inserted into the radial or femoral arteries and invasive blood pressure monitoring was done. Four procedures were performed using an electroanatomic mapping system (EnSite Velocity ${ }^{\mathrm{TM}}$, St. Jude Medical Inc., St. Paul, MN, USA) in addition to fluoroscopy, while the other 2 were performed in emergency conditions using only fluoroscopy. Electrophysiologic catheters were inserted through the femoral and internal jugular veins. In 4 procedures, 2 venous accesses (jugular and femoral vein) were obtained. The other 2 procedures, there was only 1 venous access could be obtained and esophageal pacing catheter was used as the second catheter. All patients received $100 \mathrm{U} / \mathrm{kg}$ intravenous heparin during the ablation procedure with RFA. To map the tachycardia, we attempted catheter placement in the high right atrium, His bundle region, right ventricular apex and coronary sinus. To gain access to the left atrium, patent foramen ovale (PFO) was used. A standard atrial and ventricular protocol using single and double extrastimuli and burst pacing was used to induce SVT. In most cases the tachycardia was incessant and a complete atrial and ventricular protocol could not be performed before ablation. None of the patients required isoprenaline to sustain SVT. A diagnostic study was performed to determine the electrophysiological features of the accessory atrioventricular (AV) connections, the tachycardia mechanisms, and the location of the accessory pathways.

\section{Catheter ablation techniques and post-procedure management}

In all procedures, the atrial approach was used for ablation of the arrhythmogenic substrate. Access for ablation in the right atrium was obtained through the femoral vein in 5 procedures and the jugular vein in 1 procedure. Access to the left atrium was through the PFO.

Radiofrequency ablation. A 4-mm tip $5 \mathrm{~F}$ steerable quadripolar ablation catheter (St. Jude Medical Inc., St. Paul, MN, USA) was used for RFA. Once the ablation site was determined, 40-45 W RF energy was delivered for 25-47 s (for 1 application) through the distal electrode of the ablation catheter. RF energy was delivered during sinus rhythm or SVT, or both depending on the stability of the catheter during each. Whenever application of energy terminated the SVT, or abolished ventri- 
cular pre-excitation, a second or more application (range, 2-4) was delivered at the same site with the same power.

Cryoablation. An electroanatomic mapping system was used for cryoablation. The curve of the 6-mm tip $7 \mathrm{~F}$ cryocatheter (FreezorTM, Medtronic Inc., Minneapolis, MN, USA) was not suitable for turning left after the foramen ovale and could only be used when the arrhythmogenic substrate was on the right. At first, cryomapping (for less than $30 \mathrm{~s}$ and set to a minimum temperature of $-30^{\circ}$ ) was performed to determine the area of the earliest retrograde ventriculoatrial (VA) conduction during SVT. Then, cryoablation was applied to the area at a minimum temperature of $-60^{\circ}$ for $240 \mathrm{~s}$. Whenever application of energy terminated the SVT, more was delivered to the same site for the same amount of time.

After both RFA and TCA, a period of $30 \mathrm{~min}$ was allowed and ablation was considered successful if (1) no SVT could be induced at rest or with isoprenaline, and retrograde conduction showed decremental properties with a normal septal sequence; (2) SVT could not be induced and there was no retrograde conduction; (3) ventricular pre-excitation was abolished. At the end of the procedure, all patients were returned to the intensive care unit and underwent continuous telemetry and Holter ECG monitoring for 48-72 h. Echocardiograms were taken immediately after the procedure. Aspirin (5 mg/kg) was given daily for 6 weeks for antiaggregation. A surface ECG, Holter ECG and an echocardiogram were done at follow-up.

\section{Results}

The clinical features and cardiac statuses of the patients prior to ablation are summarized in Table 1 . The average patient age was $3.3 \pm 3.9$ months (12 days -9.5 months); average weight $-5.4 \pm 2.2 \mathrm{~kg}(3.5-9 \mathrm{~kg})$. Surface ECGs of all patients showed reentrant SVT with narrow QRS. Two of the patients had structural heart anomalies while 3 had PFO. At admission since 4 patients were in cardiac shock, they were observed in the intensive care unit and intubated. Due to hypotension and metabolic acidosis, patients were started intravenous dopamine, adrenaline and milrinone. Patients had inotropic agents until the ablation procedure. Prior to the procedure, all patients received antiarrhythmic drugs including class Ic and III agents. Antiarrhythmic treatment responses had been monitored with the 24 -h Holter and telemetric monitoring. Four of the 5 patients

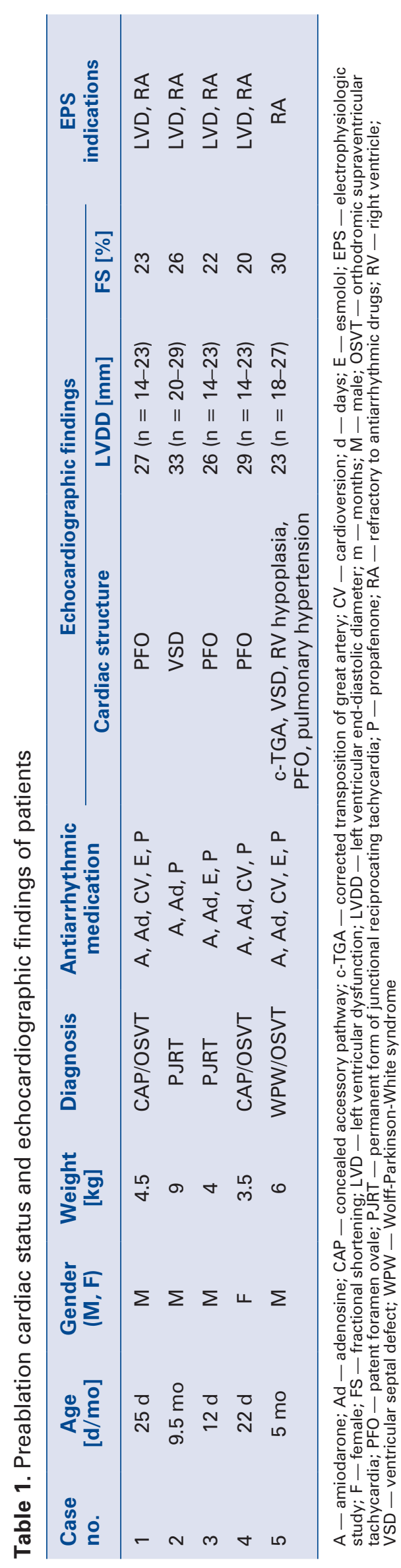


showed no response to adenosine and/or cardioversion. All patients were on intravenous amiodarone ( $5 \mathrm{mg} / \mathrm{kg}$ loading $-10 \mathrm{mg} / \mathrm{kg} /$ day maintenance). The duration of amiodarone treatment varied between $48 \mathrm{~h}-10$ days (average $95.3 \mathrm{~h}$ ). There were incessant SVT attacks $90 \%$ of day in 4 patients despite the medical treatment. The remaning 1 patient (Patient 5) had recurrent STV attacks (about $1 / 3$ of day) and arterial blood pressure had dropped 44/22 mm Hg during the attacks. In brief, electrophysiologic study and ablation were preferred since there were SVT attacks and there was request for inotropic agents despite the medical treatment for 4 patients, there were recurrent SVT attacks and clinical deterioration for 1 patient.

\section{Electrophysiological study and ablation procedure characteristics}

Table 2 summarizes the electrophysiologic study findings of patients. Two patients had a right posteroseptal accessory pathway, 2 - left posteroseptal and 1 - left lateral. There were 4 concealed and 1 manifest accessory pathways. Two of the concealed pathways had slow conduction time and decremental properties (the permanent form of junctional reciprocating tachycardia - PJRT). The tachycardia cycle length was $284 \pm 38 \mathrm{~ms}$. Except one (Patient 5), all patients had incessant tachycardia during the electrophysiology study.

The ablation data are summarized in Table 2. Patients underwent a total of 6 successful procedures. The patient with complex congenital heart disease and Wolff-Parkinson-White syndrome (WPW) (Patient 5) reverted to sinus rhythm after a successful ablation and was kept in the intensive care unit for $48 \mathrm{~h}$. However, the patient had another SVT attack 7 days after the procedure and underwent a second ablation procedure. Three patients with left-sided accessory pathways underwent RFA in the left atrium via PFO, while 2 patients with PJRT underwent TCA. An average of $3 \pm 0.8 \mathrm{RFA}$ lesions were placed in total, with an average duration of $98 \pm 38 \mathrm{~s}$. The average total time of RFA procedures (measured from the time the patient entered the laboratory until exit time) was $161 \pm$ $\pm 59 \mathrm{~min}$, while the average fluoroscopy time was $11.1 \pm 7 \mathrm{~min}$. An average of $7 \mathrm{TCA}$ applications was performed in 2 patients with PJRT, with an average ablation time of $1750 \pm 240 \mathrm{~s}$. The average total TCA procedure time (measured from the time the patient entered the laboratory until exit time) was $180 \mathrm{~min}$, while the average fluoroscopy time was $2.6 \mathrm{~min}$. Four procedures were carried out using an electroanatomic mapping system (average flu-

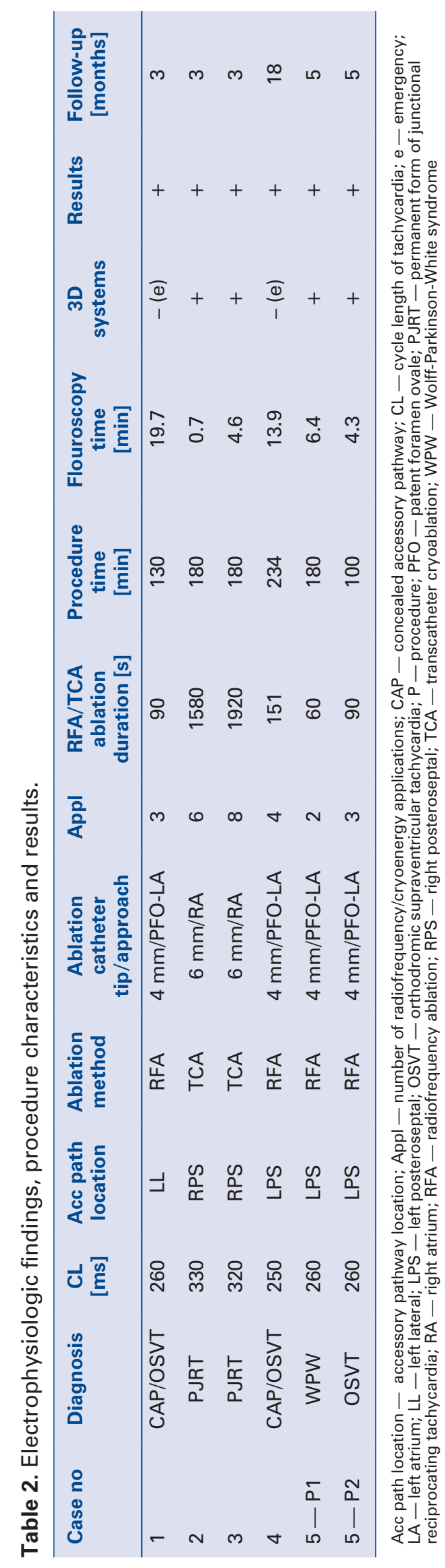


oroscopy time $4 \pm 2.4 \mathrm{~min}$ ), while 2 procedures were carried out in emergency circumstances relying on fluoroscopy (average fluoroscopy time $16.8 \pm 4.1 \mathrm{~min})$.

Following catheter ablation, all patients were evaluated using ECG, telemetry, Holter ECG and echocardiography. Patients with left ventricular dysfunction recovered completely and were gradually taken off inotropic agents. Heparin administration was continued for $24 \mathrm{~h}$ in patients with left-sided lesions. All patients were on aspirin ( $5 \mathrm{mg} / \mathrm{kg} /$ day, single dose) for 6 weeks. Echocardiography showed that Patient 3 developed moderate tricuspid valve insufficiency and hyperechogenicity in the annulus next to the valve septal leaflet following the procedure. As edema or thrombosis could not be excluded, the patient received heparin for 48 more hours and the findings had completely regressed by the time of the last examination. Average hospitalization time (pre-procedure, intensive care and hospital stay) was $12 \pm 4$ (range: 6-17) days. Echocardiographic evaluation of all patients prior to their release showed normal left ventricular function. After an average follow-up period of 6.5 (range: $3-18$ ) months, all patients were symptom free without medication.

\section{Discussion}

This report demonstrates that RFA and/or TCA can be a reliable and effective option in cases where neonates or infants have hemodynamically compromising SVT that persists despite aggressive medical treatment. The natural course of SVT in most neonates and infants includes resolution of the tachycardia by 1 year of age [1, 2]. Normally, pharmacologic therapy is used to suppress the tachycardia until spontaneous resolution occurs, but in some infants SVT cannot be controlled with a traditional first-line agent such as digoxin or propranolol. If these fail, other antiarrhythmic agents like amiodarone, flecainide, propafenone and sotalol are added or used in combination $[1,2,13]$. If SVT persists despite conventional pharmacologic therapy and is complicated by ventricular dysfunction, catheter ablation can be used for definitive treatment [2-7].

Although the number of studies focusing on the use of RFA to manage SVT in neonates and infants increases by the day, the topic remains controversial [2-8]. According to the Pediatric Radiofrequency Ablation (RFCA) registry data from 1991-1997, body weight of less than $15 \mathrm{~kg}$ poses a significant risk of complications during RFA, including AV block, cardiac perforation and even death [5]. However, a 2001 subgroup analysis of patients younger than 18 month-old (all of whom weighed under $15 \mathrm{~kg}$ ) showed no increased procedural risk relative to the rest of the database population [6]. This controversy was highlighted by the recently published North American Society of Pacing and Electrophysiology (NASPE) expert consensus conference in which the use of RFA in children younger than 5 years of age with SVT refractory to drug therapy, including sotalol and amiodarone, was believed to represent a class IIb indication [14]. Lastly, Aiyagari et al. [3] reported on the risks and results of RFA use in children under $15 \mathrm{~kg}$ and between 15 and $20 \mathrm{~kg}$, stating that RFA use in small children was safe and effective. The main reason for contradictions is that there are really limited number of RFA indications in neonates and infants with SVT and thus less experiences with these situations. Use of RFA in small children can lead to cardiac perforation, pericardial effusion, pneumothorax, coronary artery injury, AV block, and death. Additionally, the potential progressive nature of RFA lesions can also increase complication rates in younger patients [6-11]. In order to minimize or altogether avoid these complications, RFA should be performed by an experienced pediatric electrophysiologist who can reduce risk by varying power, temperature, application duration and number of attempts depending on the patient's size [6,7]. In our study, catheter ablation was carried out by an experienced pediatric electrophysiologist, and no major complications were seen. RF energy was delivered via a 4-mm tip catheter, application duration was $30 \mathrm{~s}$ on average and was always preceeded by a $3-5 \mathrm{~s}$ test application. Although some of our patients were only 12 day-old and weighed $3.5 \mathrm{~kg}$, no major complications developed. This is attributable to all patients being closely monitored prior to and following the procedure (blood pressure monitoring via an artery catheter, constant supervision by a pediatric cardiologist, periodical ECG and ECHO follow-up) and good management of their inotropic support and ventilation needs.

The complications caused by RFA in neonates and infants bring to mind the alternative energy sources used in cryoablation. TCA has been shown to be effective and reliable in pediatric patients with AV nodal reentrant tachycardia or SVT with accessory pathways $[15,16]$. However, until 2011 , studies of TCA use in neonates and infants remained very limited $[17,18]$. The only publication on this topic is an international multicenter retrospective 
study (Members of the Pediatric and Congenital Electrophysiology Society) that included $61 \mathrm{pa}-$ tients under 5 years of age and/or weighing less than $15 \mathrm{~kg}$ [12]. Those patients who had to undergo RFA due to failed TCA developed complications, while those who only underwent TCA had none. The study also found that TCA had lower success rates and higher recurrence rates. In our study, TCA was used in two patients with PJRT and no recurrences had been seen by the end of the follow-up period. Among the advantages of cryoablation are greater catheter stability, lesion reversibility, sharply demarcated lesions that preserve tissue integrity, and a low risk of thrombosis $[15,16]$. Disadvantages include a larger catheter size and less catheter steerability, which may limit its use in neonates and infants. In our opinion, production of catheters of smaller size that will allow for more manipulation would lead to cryoablation becoming the preferred choice.

Another important point of our study is the use of an electroanatomic mapping system, with the exception of emergency cases. The EnSite system guidance can be safely and effectively used as the primary catheter visualization tool during catheter ablation. Besides its advantage in 3D mapping, this system was used to reduce fluoroscopic exposure $[19,20]$. In the pediatric ablation registry, the average fluoroscopy time for ablation of SVT is $40 \mathrm{~min}$ [21]. With newborns and infants, this time can extend to up to $194 \mathrm{~min}$ $[6,7,17]$. When we used the 3D mapping system, the average time of fluoroscopy was only $4 \mathrm{~min}$. Mapping systems present an effective alternative to leaving younger patients exposed to radiation for long periods of time.

\section{Conclusions}

Catheter ablation in neonates and infants should be reserved for life-threatening arrhythmias that persist after multiple failed attempts at medical management, including various combination therapies. RFA should be performed by an experienced pediatric electrophysiologist who can use various strategies to reduce complication risks based on the patient's size. TCA can be a safe and effective option to RFA in selected patients.

Conflict of interest: none declared

\section{References}

1. Garson A, Gillette PC, McNamara D. Supraventricular tachycardia in children: clinical features, response to treatment, and long-term follow-up in 217 patients. J Pediatr, 1981; 98: 875-882.

2. Weindling SN, Saul JP, Walsh EP. Efficacy and risks of medical therapy for supraventricular tachycardia in neonates and infants. Am Heart J, 1996; 131: 66-72.

3. Aiyagari R, Saarel EV, Etheridge SP, Bradley DJ, Dick M $2^{\text {nd }}$, Fischbach PS. Radiofrequency ablation for supraventricular tachycardia in children $<$ or $=15 \mathrm{~kg}$ is safe and effective. Pediatr Cardiol, 2005; 26: 622-626.

4. Kugler JD, Danford DA, Deal BJ, et al. Radiofrequency catheter ablation for tachyarrhythmias in children and adolescents. N Engl J Med, 1994; 330: $1481-1487$.

5. Kugler JD, Danford DA, Houston K, Felix G. Radiofrequency catheter ablation for paroxysmal supraventricular tachycardia in children and adolescents without structural heart disease: Pediatric EP Society, Radiofrequency Catheter Ablation Registry. Am J Cardiol, 1997; 80: 1438-1443.

6. Blaufox AD, Felix GL, Saul JP; Pediatric Catheter Ablation Registry. Radiofrequency catheter ablation in infants $</=18$ months old: When is it done and how do they fare? Short-term data from the pediatric ablation registry. Circulation, 2001; 104: 2803-2808.

7. Blaufox AD, Paul T, Saul JP. Radiofrequency catheter ablation in small children: relationship of complications to application dose. Pacing Clin Electrophysiol, 2004; 27: 224-229.

8. Schaffer MS, Gow RM, Moak JP, Saul JP. Mortality following radiofrequency catheter ablation (from the Pediatric Radiofrequency Ablation Registry). Participating members of the Pediatric Electrophysiology Society. Am J Cardiol, 2000; 86: 639-643.

9. Erickson CC, Walsh EP, Triedman JK, Saul JP. Efficacy and safety of radiofrequency ablation in infants and young children less than eighteen months of age. Am J Cardiol, 1994; 74: 944-947.

10. Saul JP, Hulse JE, Walsh EP. Late enlargement of radiofrequency lesions in infant lambs: Implications for ablation procedures in small children. Circulation, 1994; 90: 492-499.

11. Paul T, Kakavand B, Blaufox AD, Saul JP. Complete occlusion of the left circumflex coronary artery after radiofrequency catheter ablation in an infant. J Cardiovasc Electrophysiol, 2003; 14: 1004-1006.

12. LaPage MJ, Reed JH, Collins KK, et al. Safety and results of cryoablation in patients $<5$ years old and/or < 15 kilograms. Am J Cardiol, 2011; 108: 565-571.

13. Price JF, Kertesz NJ, Snyder CS, Friedman RA, Fenrich AL. Flecainide and sotalol: a new combination therapy for refractory supraventricular tachycardia in children < 1 year of age. J Am Coll Cardiol, 2002; 39: 517-520.

14. Friedman RA, Walsh EP, Silka MJ et al. NASPE Expert Consensus Conference: Radiofrequency catheter ablation in children with and without congenital heart disease: Report of the writing committee: North American Society of Pacing and Electrophysiology. Pacing Clin Electrophysiol, 2002; 25: $1000-1017$

15. Tuzcu V. Cryoablation of accessory pathways in children. Pacing Clin Electrophysiol, 2007; 30: 1129-1135.

16. Bar-Cohen Y, Cecchin F, Alexander ME, Berul CI, Triedman JK, Walsh EP Cryoablation for accessory pathways located near normal conduction tissues or within the coronary venous system in children and young adults. Heart Rhythm, 2006; 3: 253-258.

17. Shah MJ, Wieand T, Vetter VL. Cryoablation of congenital familial ectopic tachycardia with preservation of atrioventricular nodal function in an infant. J Cardiovasc Electrophysiol, 2007; 18: 773-776.

18. Makhoul M, Von Bergen NH, Rabi F, Gingerich J, Evans WN, Law IH. Successful transcatheter cryoablation in infants with drug-resistant supraventricular tachycardia: A case series. J Interv Card Electrophysiol, 2010; 29: 209-215.

19. Tuzcu V. A nonfluoroscopic approach for electrophysiology and catheter ablation procedures using a three-dimensional navigation system. Pacing Clin Electrophysiol, 2007; 30: 519-525.

20. Smith G, Clark JM. Elimination of fluoroscopy use in a pediatric electrophysiology laboratory utilizing three-dimensional mapping. Pacing Clin Electrophysiol, 2007; 30: 510-518.

21. Kugler JD, Danford DA, Houston KA, Felix G. Pediatric radiofrequency catheter ablation registry success, fluoroscopy time, and complication rate for supraventricular tachycardia: Comparison of early and recent eras. J Cardiovasc Electrophysiol, 2002; 13: 336-341. 